Vol. 8, Issue 8, August 2021

DOI: 10.17148/IARJSET.2021.88112

\title{
Designing pollen inspired OPH enclosed micro particles for reducing insecticide toxicity in Bombusimpatiens ( Bumblebees) : A review
}

\author{
Amruta Kumbhar $^{1}$, Jyoti Dubey ${ }^{2}$, Akash Patil $^{3}$, Tanvi Patil ${ }^{4}$ \\ SIES College Of Arts, Science \& Commerce, Mumbai University ${ }^{1-4}$
}

\begin{abstract}
Honey bee (Apis) is an important pillar of ecosystem. They act as primary pollinators for more than $85 \%$ of food crops, herbaceous trees and small bushes. Majority of forest ecosystem is dependent upon seeds,berries and fruits which is possible because of pollination carried out by the bees. Honey bee is an inevitably important part of the ecosystem and to maintain that harmony, our primary pollinators must be prevented from the toxicity of today's chemical pesticides. Honeybees exposure to either lethal or sublethal dosage of these pesticides can directly impact global food security. During crop pollination, primary pollinators can be exposed to various harmful pesticides.This review highlights the construction of pollen inspired microparticles enclosed with enzyme Organohosphodiesterase hydrolase $(\mathrm{OPH})^{[4][10]}$. The microparticle was designed to be $\mathrm{pH}$ inducible so that it gets activated only in the digestive system of honeybees and nowhere else. The microparticle was stable and loading efficiency was found to be around $90 \%$. Microcolonies of Bombus impatiens were used for in vivo testing. It was observed that bumblebee which was fed with OPH-PIM (Organophosphodiesterase-hydrolase-Pollen Inspired Microparticle)survived where as the one fed with just pesticides and sugar died within 4-5 days. This study can further help us to explore different ways by which such studies can be applied for agricultural purpose.
\end{abstract}

Keywords- Organophosphates, microcolonies, microparticles, ecosystem, lethal dose, sublethal dose, pesticides.

\section{INTRODUCTION}

Pollination is one of the most important ecological services provided by pollinators. Honey bee (Apis) is an important pillar ofecosystem. Without honeybees, as it's already known to us and we have been warned as well that human life would be impossible. Honey bee inevitably is an important part of the ecosystem and to maintain that harmony, our primary pollinators must be prevented from the toxicity of modern chemical pesticides. We loose around $30 \%$ of our bees each year and despite all warnings, the number still keeps on increasing due to lack of action and awareness among people and also due to lack of resources to overcome this issue which ultimately threatens our food security $[7][8][9]$.

During crop pollination, primary pollinators can be exposed to various harmful pesticides. Exposure to lethal or sublethal doses of pesticides like organophosphates can impair the fitness of wild as well as managed bees hence risking the pollination quality and food security as well. OP insecticides influence insects cholinergic neural signalling through inhibition of carboxyl ester hydrolases mainly the Acetylcholine esterases (AchE) which breaks down acetylcholine. OPs inactivate AchE via irreversible covalent inhibition causing buildup of the acetylcholine and hence overstimulation of nicotinic and muscarinic receptors ${ }^{[1]}$. LD50 of malathion and Parathion is 0.38 and $0.175 \mathrm{ug} / \mathrm{bee}$. It is understood that organophosphates are the main pesticides which are affecting honeybees specially Malathion and Parathion. According to a study published in Nature food, designing a microparticle and enclosingPhosphotriesteraseenzyme which can degrade these harmful pesticides can be a real deal as it can degrade the pesticides in bees digestive system before pesticides are digested completely and begin to cause any harmful effect on bees. Phosphotriesterases are metallozymes which causes hydrolysis of thetriester linkage found in OP insecticides. Both the bumble bee and the honey bee have a GI tract consisting of a crop and ventriculus separated by proventricular valve which mechanistically extracts out the micron sized particles for the purpose of digestion. The $\mathrm{pH}$ of crop and ventriculus in honeybee is 4.8 and 6.5 respectively ${ }^{[5]}$. In this study, a pollen InspiredMicroparticle (PIMs) enclosedwith the enzyme Organophosphate- Phosphodiesterase (Hydrolase) was designed.The microparticle was designed to be $\mathrm{pH}$ inducible so that it gets activated only in the digestive system of the honeybees. Such microparticles can be fed to the bees by suspending it in sugar syrups or pollen patties ${ }^{[3]}$. 
International Advanced Research Journal in Science, Engineering and Technology

Vol. 8, Issue 8, August 2021

DOI: 10.17148/IARJSET.2021.88112

METHODOLOGY

In this particular study, Bombus impatiens (bumble bees) were taken for the experimentation purpose. However, as they are closely related to honeybees, the results are also applicable for Apismellifera. In order to design $\mathrm{pH}$ inducible OPH enclosed PIMs, first enzyme (Organophosphate hydrolases) is isolated and purified. The most commonly used enzyme is Amidohydrolase phosphotriesterase obtained from the bacterium Pseudomonas diminuta Or Flavobacterium ATCC $27551^{[2][6]}$.It also degrades thiol linkage in malathion. OPH has poor stability in low $\mathrm{pH}$ and high temperature. At the $\mathrm{pH}$ range of 8.0-9.5, the $\mathrm{OPH}$ can tolerate thermostability upto $45^{\circ} \mathrm{C}$, but gets degraded at temperature above $60^{\circ} \mathrm{C}$. It can be isolated from nature or direct synthetic preparation can also be utilized. After getting the purified enzyme form, we need to store it at $4^{\circ} \mathrm{C}$ to prevent any aggregation.

When it comes to the second part of the experiment, we need to prepare calcium carbonate microparticles, for that $0.33 \mathrm{M}$ volume of the $\mathrm{CaCl}_{2}$ mixed with $\mathrm{NaCO}_{3}$. Various parameters like stirring speed, time taken for stirring, additive inclusion should be taken into consideration to get a microparticle of uniform size and shape. Scanning Electron Microscopy (SEM) can be employed to observe the pore size of the microparticle. Microparticle pore size can also be analyzed in accordance with the density functional theory using $\mathrm{N}$ absorption isotherms. The microparticle must be having aggregated structure which can act as porous accessible channel for the purpose of biomacromolecule diffusion. Biophotometer device can be used to understand the suspension stability of these microparticles in sucrose. The OPH was enclosed within the microparticle via sonication and protein loading efficiency (PLE) was determined.

In the third part of our study, in vivo analysis regarding the activity of the microparticle was carried out using 2 sets of honeybees. One set, considered as test set and another as negative control set. The test was fed with $0.5 \mathrm{mM}$ paraoxon and $0.44 \mathrm{mM}$ of malathion along with $\mathrm{OPH}$ enclosed microparticle suspended in sugar syrup or pollen patties. Whereas, the control was fed with $0.5 \mathrm{mM}$ paraoxon and $0.44 \mathrm{mM}$ malathion and plain sugar syrup. The observation was carried out for 4-5 days. Later on, the degradation of paraoxon was understood by carrying out paraoxon assays where production of free nitrophenol shows proper activity of PIMs. Malathion hydrolysis can be understood by using Ellmans reagent.

\section{RESULTS}

For the 1st part of the study, calcium carbonate microparticle was designed and the gelatin was employed as an additive. Size of the microparticle was around 3-4 um. The stirring time of 10 seconds was found to be suitable for proper size. Biophotometer was used to measure the suspension stability of the PIMs in sucrose and it was observed that PIMs took 6 days to fully settle down. The amount of sucrose for suspension was taken to be $2 \mathrm{~g} / \mathrm{ml}$. The shelf life of the OPH microparticle was also determined and it is thought to be stable at $4{ }^{\circ} \mathrm{C}$ other wise activity reduces after 2 weeks. The microparticle showed $60 \%$ activity after 7 days and $49.5 \%$ activity after 14 days. Scanning Electron microscopy was carried out to know the overall size of the microparticle. The surface area was found to be very high which is suitable for the physical adsorption and high substrate loading. Through in vivo analysis, it was shown that, the test had $100 \%$ survival rate whereas most of the negative control bees died within 4 days. Absorbance from malathion hydrolysis was characterized using Ellman's ( 5,5'-dithiol -(2-nitrobenzoic acid) or DTNB) reagent which reacted with the thiol group of malathion after break down to form product called 2-Nitro-5-thiobenzoate . The absorbance was measured at $412 \mathrm{~nm}$. During 1 st hour of digestion, microparticles distributed across both the crop stomach and ventriculus. By the 4 hours, most of the microparticle had travelled out of crop stomach before full clearance. After 12 hours, the microparticle was found to be present in the ventriculus which suggested proventricular filtering of the PIMs. Further, the AchE assay was carried out to get a clear idea whether microparticle works or not as the major impact of the pesticide is on the AchEactivity.AchE activity can be quantified by understanding acetylthiocholine cleavage via AchE. Thiocholine interacts with the DTNB to form TNB2 whose absorbance maxima is at $412 \mathrm{~nm}$. It was observed that the honey bees could maintain $91.5 \%$ of their AchE activity after going treatment with. 5mM paraxon and OPHPIMs. This was a stark improvement in AChE functionality relative to no treatment, which resulted in a relative activity reduction of $\sim 72 \%$.Increase in the AchE activity shows effective detoxification.

\section{CONCLUSION}

As the pollen grains progresses through the mid gut,it releases its internal contents. Through this experiment , it is understood that PIMs enhanced the bioactivity of the OPH. The OPH-PIMs outperformed free OPH when tested under unfavorable conditions if adverse temperature and storage. It bestows functionality in gastric acidity and can be maintained for longer duration under thermal stress. There is appreciable benefit when degrading OPs which are hydrolyzed at lower rate. This work has resulted in a viable product which can be helpful in mitigating insecticide damage to pollinator colonies and also has revealed us new ways by which this technique can be enhanced. Hence, we can conclude that PIMs with enclosed OPH can become a revolutionary technique by which we will be able to reduce 


\section{International Advanced Research Journal in Science, Engineering and Technology}

Vol. 8, Issue 8, August 2021

\section{DOI: 10.17148/IARJSET.2021.88112}

the pesticide toxicity in honey bees. Further research need to be carried out in order to determine impact of this unique technique on a colony as whole that is colony scale testing need to be carried out. If it is to be applied at a large scale for agricultural purpose, we need to comprehend the economic potential of the design before hand.

\section{REFERENCES}

[1] Fukuto, T. R. Mechanism of action of organophosphorus and carbamate insecticides. Environ. Health Perspect. 87, 245-254 (1990).

[2] Caldwell, S. R., Newcomb, J. R., Schlecht, K. A. \&Raushel, F. M. Limits of diffusion in the hydrolysis of substrates by the phosphotriesterase from Pseudomonas diminuta. Biochemistry 30, 7438-7444 (1991).

[3] Chen, J., Webb, J., Shariati, K. et al. Pollen-inspired enzymatic microparticles to reduce organophosphate toxicity in managed pollinators. Nat Food 2, 339-347 (2021).

[4] Singh, B. K. Organophosphorus-degrading bacteria: ecology and industrial applications. Nat. Rev. Microbiol. 7, 156-164 (2009).

[5] PENG, Y. S. \& MARSTON, J. M. Filtering mechanism of the honey bee proventriculus. Physiol. Entomol. 11, 433-439 (1986).

[6] Naqvi, T. et al. A 5000-Fold Increase in the Specificity of a Bacterial Phosphotriesterase for Malathion through Combinatorial Active Site Mutagenesis. PLoS One 9, 7 (2014).

[7] Sanchez-Bayo, F. \&Goka, K. Pesticide Residues and Bees - A Risk Assessment. PLoS One 9, 16 (2014).

[8] Croft, B. A. Arthropod biological control agents and pesticides. John Wiley and Sons Inc. (1990).

[9] Reilly, J. R. et al. Crop production in the USA is frequently limited by a lack of pollinators. Proc. R. Soc. B-Biol. Sci. 287, 8 (2020).

[10] McLoughlin, S. Y., Jackson, C., Liu, J. W. \& Ollis, D. Increased expression of a bacterial phosphotriesterase in Escheri chia coli through directed evolution. Protein Expr. Purif. 41, 433-440 (2005).

[11] Pooja Dahiya; Roopsi Kaushik; Anil Sindhu. "An Introduction to Plant Growth Promoting Rhizobacteria, Antifungal Metaboli tes Biosynthesis using PRPR with reference to Pseudomonas species and It's other characteristics like Antagonistic and Biocontrolling properties". International Research Journal on Advanced Science Hub, 2, Special Issue ICAMET 10S, 2020, 95-100. doi: 10.47392/irjash.2020.205 